\title{
On the Connection between RNAi and Heterochromatin at Centromeres
}

\author{
E. LEJEUne, ${ }^{1}$ E.H. BAYNe, ${ }^{1,2}$ AND R.C. AllshiRE \\ Wellcome Trust Centre for Cell Biology and Institute of Cell Biology, School of Biological Sciences, \\ The University of Edinburgh, Edinburgh EH9 3JR, Scotland, United Kingdom \\ Correspondence: robin.allshire@ed.ac.uk
}

\begin{abstract}
RNA interference (RNAi) is a conserved silencing mechanism whereby double-strand RNA induces specific down-regulation of homologous sequences. In the fission yeast Schizosaccharomyces pombe, centromeric heterochromatin assembly is an RNAi-dependent process. Noncoding RNAs transcribed from pericentromeric repeat sequences are processed into short interfering RNAs (siRNAs) that direct the Argonaute-containing RNA-induced transcriptional silencing (RITS) effector complex to homologous nascent transcripts. RITS is required for H3K9 methylation by the histone methyltransferase (HMT) Clr4; conversely, H3K9 methylation can attract RITS to chromatin via binding of the chromodomain protein Chp1. This codependency has hampered dissection of the order of events and mechanisms of cross talk between the RNAi and chromatin modification machineries. To tackle this problem, we have developed systems that reconstitute heterochromatin at a euchromatic locus, using either hairpin triggers or DNA-tethered chromatin-modifying complexes. These systems reveal that RNAi is sufficient to promote heterochromatin assembly in cis and that direct recruitment of the HMT Clr4 can bypass the role of RNAi in heterochromatin assembly. We have also characterized a new pathway component, Stc1, that translates the RNAi signal into chromatin marks. We discuss the implications of these findings for our understanding of the mechanism and function of RNAi-directed heterochromatin assembly at centromeres.
\end{abstract}

In eukaryotes, vast lengths of genomic DNA are organized within the tiny volume of the cell nucleus through packaging with proteins to form a structure called chromatin. Two types of chromatin are visible in interphase cells (Passarge 1979): (1) euchromatin, largely decondensed and gene rich, and (2) heterochromatin, tightly compacted, gene poor, and mainly composed of repetitive sequences. Constitutive heterochromatin forms at critical cellular structures such as centromeres, telomeres, and regulatory loci. It is characteristically associated with transcriptional silencing of associated genes and contributes to genome stability in various ways, including promoting proper centromere function, nuclear organization, and suppressing transposable elements (Pidoux and Allshire 2005; Grewal and Jia 2007).

Studies across eukaryotes have contributed to our understanding of heterochromatin and revealed that enzymes and structural components involved in heterochromatin formation are conserved from fission yeast to humans (Grewal and Elgin 2007). The minimal unit of chromatin is the nucleosome, which is composed of $\sim 150 \mathrm{bp}$ of DNA wrapped around an octamer of histone proteins $\mathrm{H} 2 \mathrm{~A}, \mathrm{H} 2 \mathrm{~B}, \mathrm{H} 3$, and H4 (Luger et al. 1997). The amino-terminal tails of histones are subject to various posttranslational modifications including acetylation and methylation that, in turn, define docking sites for structural factors (Jenuwein and Allis 2001). Whereas euchromatin is associated with hyperacet-

\footnotetext{
${ }^{1}$ These authors contributed equally.

${ }^{2}$ Present address: Wellcome Trust Centre for Gene Regulation and Expression, College of Life Sciences, University of Dundee, Dundee DD1 5EH, Scotland, United Kingdom.
}

ylation, heterochromatic domains are generally hypoacetylated and characteristically marked by methylation of histone $\mathrm{H} 3$ on lysine 9 (H3K9me), which provides a specific binding site for the HP1 family of chromodomain proteins (Bannister et al. 2001; Jenuwein and Allis 2001; Lachner et al. 2001). The enzymes that catalyze histone modifications together with the factors that target those marks are integral interacting components of heterochromatin, and iterated cycles of docking to, and modifying of, histone tails lead to spreading of the heterochromatin domain along the chromatin fiber (Nakayama et al. 2001).

The mechanisms that contribute to heterochromatin assembly are best characterized in the fission yeast $S$. pombe (Grewal and Jia 2007). Analogous to those in higher eukaryotes, $S$. pombe centromeres comprise central domain DNA where the histone H3 variant CENP-A ${ }^{\text {cnp1 }}$ is deposited and the kinetochore assembles, flanked by tandem and inverted arrays of repetitive elements ( $d g$ and $d h$ ) that are assembled in heterochromatin (Fig. 1) (Pidoux and Allshire 2005). Almost 10 years ago, it was discovered that components of the RNAi pathway are involved in directing heterochromatin assembly on these centromeric outer-repeat sequences (Volpe et al. 2002). Since then, detailed analyses have shed light on the underlying mechanism of this multistep and self-reinforcing process. RNAi is a conserved eukaryotic cellular pathway whereby double-strand RNA molecules induce specific repression (silencing) of genes with complementary sequences (Hannon 2002). This silencing can occur at the transcriptional or posttranscriptional level; in $S$. pombe, RNAi appears to function primarily in transcriptional silencing via histone modification. 

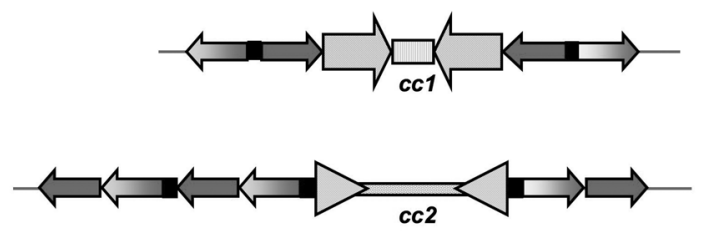

cc2

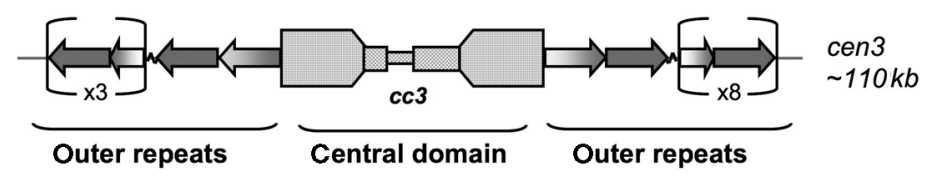

cen1

$\sim 40 \mathrm{~kb}$

cen2

$\sim 65 \mathrm{~kb}$

Figure 1. Fission yeast centromeres. The fission yeast genome is spread over three chromosomes, each having a unique centromere organization ranging in size from $\sim 40$ to $\sim 110 \mathrm{~kb}$. Heterochromatin coats the arrays of outer-repeat sequences, whereas the histone $\mathrm{H} 3$ variant $\mathrm{CENP}-\mathrm{A}^{\text {cnp } 1}$ localizes on the central domain where the kinetochore assembles. (cc) Central core.
The centromeric outer-repeat sequences in $S$. pombe are transcribed during $\mathrm{S}$ phase by RNA polymerase II (Pol II), giving rise to noncoding RNAs that are engaged by the RNAi machinery (Chen et al. 2008; Kloc et al. 2008; Zhang et al. 2008). The RNase III-like ribonuclease Dicer (Dcr1) cleaves centromeric double-strand RNA (dsRNA) into siRNAs. These siRNAs are bound by the Argonaute protein Ago1, part of the effector complex RITS (comprising Ago1, Chp1, and Tas3), and target RITS to cognate chromatin, probably via base pairing with complementary nascent transcripts (Fig. 2) (Verdel et al. 2004). RITS recruits the RNA-dependent RNA polymerase complex (RDRC) (Rdp1, Cid12, and Hrr1) to chromatin that, in turn, promotes further dsRNA production, generating a positive-feedback loop (Motamedi et al. 2004; Colmenares et al. 2007). A subset of splicing factors, possibly associated with transcribing RNA Pol II, contributes to this amplification cycle (Bayne et al. 2008). Ultimately, RNAi activity results in the recruitment of Clr4, the homolog of human Suv39h, and the only H3K9 HMT in $S$. pombe. Clr4 associates with Rik1, Dos1, Dos2, and Cul4 proteins in a complex (CLRC) that exhibits cullin-dependent E3 ubiquitin ligase activity (Bannister et al. 2001; Nakayama et al. 2001; Sadaie et al. 2004; Hong et al. 2005; Horn et al. 2005; Jia et al. 2005; Li et al. 2005; Zhang et al. 2008). Although the relationship between the ubiquitin ligase activity of CLRC and the HMT activity of Clr4 is unclear, all members of the CLRC complex are required for heterochromatin assembly. Clr4-mediated H3K9 methylation creates binding sites for four $S$. pombe chromodomain proteins: Swi6, Chp1, Chp2, and Clr4 (Bannister et al. 2001; Sadaie et al. 2004; Zhang et al. 2008). Binding of Swi6 allows recruitment of cohesin, whereas binding of Chp 1 and Clr4 can stabilize the chromatin interactions of RITS and CLRC, respectively, reinforcing the targeting signal and promoting the spreading of heterochromatin.

The RNAi machinery has a critical role in driving heterochromatin assembly on centromeric repeats. However, feedback of H3K9me on RNAi is also clearly important;

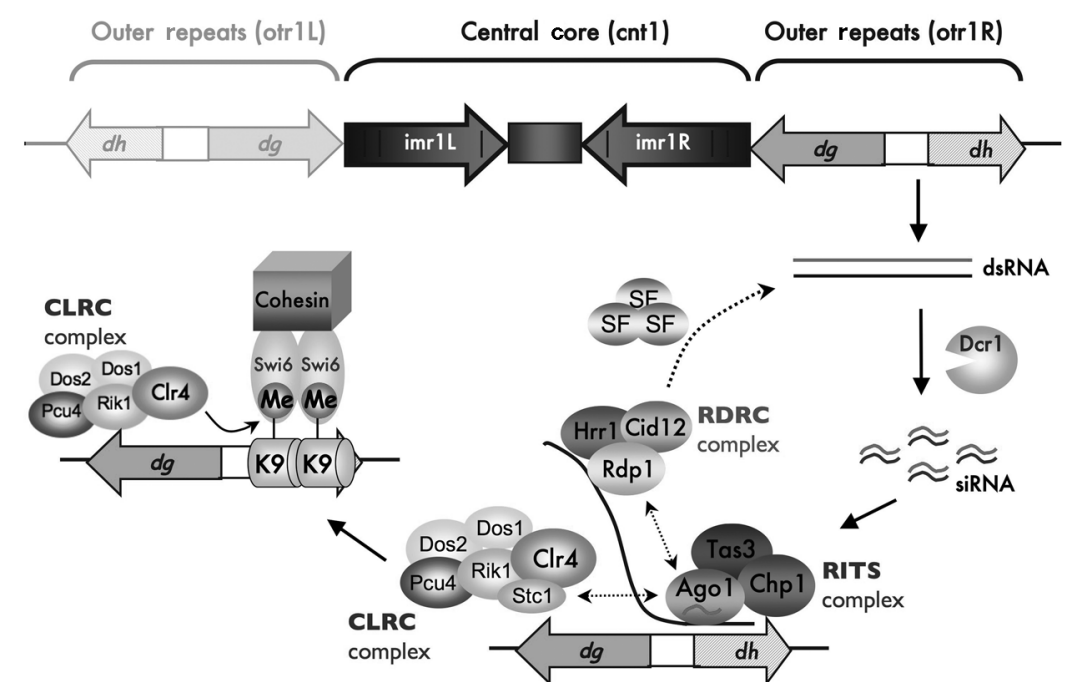

Figure 2. Model for RNAi-directed heterochromatin formation. Transcription of the centromere outer repeats (otrs) generates dsRNA, which can be processed into siRNAs by the RNase III-like enzyme Dcr1. The siRNAs are loaded into Ago1, targeting the effector complex RITS to the otr region by a base-pairing mechanism. RITS interacts with the RDRC complex, increasing the pool of siRNAs via Rdp1 RNA-dependent RNA polymerase activity. A subset of splicing factors also contributes to this amplification process. The activity of the RNAi machinery results in recruitment of the CLRC histone-modifier complex to chromatin, allowing the CLRC subunit $\mathrm{Clr} 4$ to methylate histone $\mathrm{H} 3$ on lysine 9. This creates a mark that is recognized by the chromodomain protein Swi6 that, in turn, recruits cohesin, which is required for proper biorientation of centromeres during mitosis. 
for example, whereas deletion of the RNAi component Dcr1 leads to a decrease in H3K9 methylation at centromeres, deletion of the HMT Clr4 likewise causes a severe reduction in siRNA accumulation. Thus, in either case, both the heterochromatin-based docking platform and the RNAi-mediated processing of centromeric transcripts are disrupted (Zhang et al. 2008). This interdependency indicates that heterochromatin is maintained via a positive-feedback loop, making it difficult to discern the linear order of events that lead to heterochromatin assembly. One way to overcome this problem is to examine the processes leading to de novo establishment of heterochromatin, which can reveal facets of the pathway that are masked when studying maintenance of preexisting H3K9me. For example, although knocking out the RNAi pathway has only a modest effect on maintenance of $\mathrm{H} 3 \mathrm{~K} 9$ me at centromeres, when $\mathrm{H} 3 \mathrm{~K} 9 \mathrm{me}$ is erased by transient removal of Clr4, RNAi is absolutely required to reestablish centromeric H3K9me (Sadaie et al. 2004). Similarly, at the silent mating-type locus, RNAi is dispensable for maintenance of heterochromatin because of the action of a redundant pathway involving DNA-binding proteins Atf1/Pcr1. However, removal of preexisting $\mathrm{H} 3 \mathrm{~K} 9 m$ me reveals that RNAi is essential to nucleate heterochromatin formation at this locus (Jia et al. 2004; Kanoh et al. 2005; Hansen et al. 2006). Clearly, although maintenance of heterochromatin naturally follows a preceding establishment phase, the molecular mechanisms underlying those processes can be distinct. Therefore, when seeking to understand the relationship between $\mathrm{H} 3 \mathrm{~K} 9 \mathrm{me}$ and RNAi in heterochromatin formation, artificial systems amenable to the study of both establishment and subsequent maintenance steps provide valuable tools.

To define the requirements for heterochromatin assembly at centromeres, we have developed assays that recapitulate this phenomenon by either (1) using a hairpin to trigger RNAi against a transgene at a euchromatic locus or (2) tethering the HMT Clr4 directly to a euchromatic reporter gene. We have also exploited the latter tool in analyzing the function of a novel component, Stc1, which appears to have a key role in connecting RNAi processing with chromatin modification. In this review, we discuss our recent progress in understanding the mechanisms of heterochromatin assembly and their relationship with the RNAi machinery.

\section{HAIRPINS AS INDUCERS OF HETEROCHROMATIN}

In most eukaryotes tested, introduction of an artificial source of siRNAs is sufficient to trigger posttranscriptional silencing of any homologous locus (Ghildiyal and Zamore 2009). Indeed, the ability to act in trans is a key feature of many RNAi-related pathways (Bartel 2009). In plants, artificial hairpin-generated siRNAs can mediate transcriptional silencing via DNA methylation and histone modification in cognate chromatin (Kanno et al. 2004; Matzke et al. 2009). We have exploited hairpin systems to test whether RNAi is sufficient to initiate de novo heterochromatin formation in fission yeast.
We and other investigators have explored the extent to which hairpins can trigger trans silencing and heterochromatin formation at homologous sites in otherwise wildtype cells (Iida et al. 2008; Simmer et al. 2010). Initially, we constructed a hairpin complementary to $200 \mathrm{bp}$ of $\mathrm{ura}^{+}$, integrated at the ars 1 locus (Fig. 3A). This hairpin generated detectable ura 4 siRNAs; however, these were not sufficient to silence the $\mathrm{ura}^{+}$gene at its endogenous locus (Simmer et al. 2010). Nevertheless, the same hairpin did induce silencing of a $\mathrm{ura}^{+}$gene inserted close to pericentromeric repeats (Simmer et al. 2010). At this site,

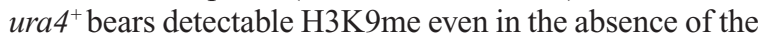
hairpin, although this is not associated with gene silencing (Allshire et al.1995). These results indicate that effective silencing may depend on the chromosomal context of the target locus and that a certain threshold of repression should be met before effective gene silencing is achieved.

We went on to test silencing via an alternative hairpin comprising the full-length green fluorescent protein (GFP) sequence (Fig. 3B). This hairpin had previously been shown to induce posttranscriptional silencing of a highly expressed target gene (Sigova et al. 2004); however, we tested its ability to silence a more moderately expressed target that we hypothesized may be more amenable to heterochromatin formation. Consistent with this idea, we found that the GFP hairpin integrated at the ars 1 locus did trigger silencing of a $\mathrm{ura}^{+}-G F P$ fusion target gene, expressed from the ura 4 promoter (Simmer et al. 2010). Silencing was dependent on the RNAi machinery and,

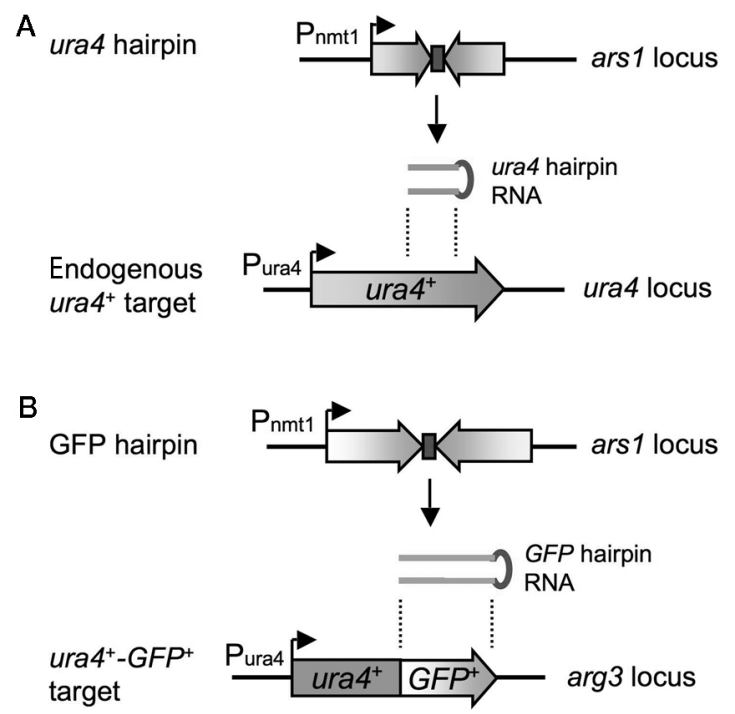

Figure 3. Artificial hairpins. (A) The ura 4 hairpin construct is composed of nucleotides 442-641 of the $\mathrm{ura}^{+}$open reading frame (ORF), arranged in inverted orientation around a 62-bp spacer corresponding to the first intron from the $\mathrm{rad}^{+}$gene and inserted at the ars 1 locus. The resulting hairpin RNA is processed into siRNAs that can target the corresponding region of the endogenous $\mathrm{ura}^{+}$gene. $(B)$ The green fluorescent protein (GFP) hairpin construct is composed of two full-length copies of the GFP ORF arranged around the rad 9 intron and inserted at the ars 1 locus. siRNAs derived from this hairpin RNA can target the GFP portion of the $\mathrm{ura}^{+}$- $G F P^{+}$target gene, which is integrated at the arg 3 locus. (Pnmt1) nmt promoter, (Pura4) ura4 promoter. 
importantly, correlated with the appearance of $\mathrm{H} 3 \mathrm{~K} 9 \mathrm{me} 2$

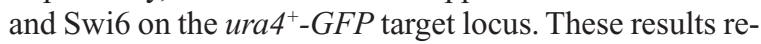
veal that hairpin-derived siRNAs are sufficient to trigger heterochromatin formation at a cognate euchromatic locus. However, this process is still relatively inefficient: Despite loading of abundant GFP siRNAs into Agol and the presence of $\mathrm{H} 3 \mathrm{~K} 9 \mathrm{me}$ on the $u r a 4^{+}-G F P$ locus, GFP transcript levels remained high (Simmer et al. 2010). In fact, greater levels of $\mathrm{H} 3 \mathrm{~K} 9 \mathrm{me} 2$ were found on the hairpin locus than on the target gene, consistent with the idea that RNAi-based H3K9 methylation operates most efficiently in cis in fission yeast. It could be that the RNAi machinery is cis restricted due to anchoring of components, such as Chp1/RITS, to chromatin at the site of dsRNA production. Indeed, several studies have found that the fission yeast RNAi machinery does not mediate efficient trans silencing, suggesting it is subject to greater constraints than in many other systems. For example, Bühler and coworkers (2006) have shown that siRNAs derived from a heterochromatic marker gene can occasionally mediate silencing of a second copy of the same gene only if the nuclease Eri1 is deleted. Similarly, hairpin constructs have been found to mediate heterochromatin formation at target loci only under certain circumstances, namely, when the target locus is bidirectionally transcribed and the HP1 protein Swi6 is overexpressed (Iida et al. 2008).

In addition to siRNA-mediated initiation of heterochromatin, the hairpin system also allowed us to investigate another unexplored aspect of RNAi in fission yeast: "transitivity." This process has been observed in plants and worms and refers to the phenomenon whereby "primary" siRNAs targeted against one part of a gene promote the generation of "secondary" siRNAs homologous to regions 3' or $5^{\prime}$ of the initial siRNA target (Sijen et al. 2001; Vaistij et al. 2002). Transitivity requires the activity of RNA-dependent RNA polymerases (RdRP) present in plants, worms, and fungi (but not mammals). In our system (the GFP hairpin/ura $4^{+}$-GFP target), deep sequencing revealed accumulation of siRNAs corresponding not only to $G F P$, but also to the ura 4 coding region and $3^{\prime}$-untranslated region, indicating secondary siRNA production on the target locus (Simmer et al. 2010). The majority of secondary siRNAs were $5^{\prime}$ of the region targeted by the hairpin, suggesting that, similar to nematodes, transitivity occurs primarily in the $3^{\prime}$ to $5^{\prime}$ direction. As expected, this transitivity was dependent on the fission yeast RNA-dependent RNA polymerase homolog Rdp1 (Simmer et al. 2010).

Several mechanisms for transitivity have been postulated. In some cases in plants, optimal secondary siRNA production requires Ago-mediated cleavage of target RNA, presumably making it a suitable substrate for RdRP activity (Allen et al. 2005; Axtell et al. 2006). Primary siRNAs have also been proposed to act as primers for RdRPs, and there is evidence for this in cases of unidirectional $\left(3^{\prime}-5^{\prime}\right)$ secondary siRNA production in plants (Moissiard et al. 2007). In Caenorhabditis elegans, RdRPs are thought to synthesize secondary siRNAs on the target template in a cleavage- and primer-independent manner; this is supported by the fact that worm secondary siRNAs bear $5^{\prime}$ triphosphates and are predominantly complementary to the target RNA (Pak and Fire 2007; Sijen et al. 2007). How secondary siRNAs are produced in fission yeast remains unclear. We found similar levels of sense and antisense secondary siRNAs corresponding to $u r a 4^{+}-G F P$, suggesting a dsRNA intermediate (Simmer et al. 2010). Moreover, all detectable centromeric siRNAs have a $5^{\prime}$ monophosphate, consistent with Dcr1-mediated cleavage, as opposed to the $5^{\prime}$ triphosphate associated with primary RdRP products (Djupedal et al. 2009). Rdp1 has been shown to function in a primer-independent manner in vitro (Sugiyama et al. 2005), and inefficient posttranscriptional silencing in $S$. pombe also argues against a requirement for Ago1-mediated transcript cleavage to prime Rdp1 activity. Together, these observations suggest that Ago 1 binding to target transcripts may be sufficient to recruit Rdp1 to mediate primerindependent second-strand synthesis, creating full-length dsRNA molecules that are then cleaved by associated Dcr1 (Fig. 4) (Colmenares et al. 2007).

The use of these artificial siRNA triggers has revealed details of events in heterochromatin assembly downstream from siRNA production. They provide a demonstration that siRNAs are sufficient to induce de novo H3K9 methylation at a euchromatic locus but that this process is efficient only in cis; trans silencing is clearly restricted in $S$. pombe. The results also show that an initial pool of siRNAs can trigger Rdp1-dependent production of secondary siRNAs corresponding to sequences outwith the initial target; this spreading of RNAi could increase the efficiency of heterochromatin propagation along the chromatin fiber.

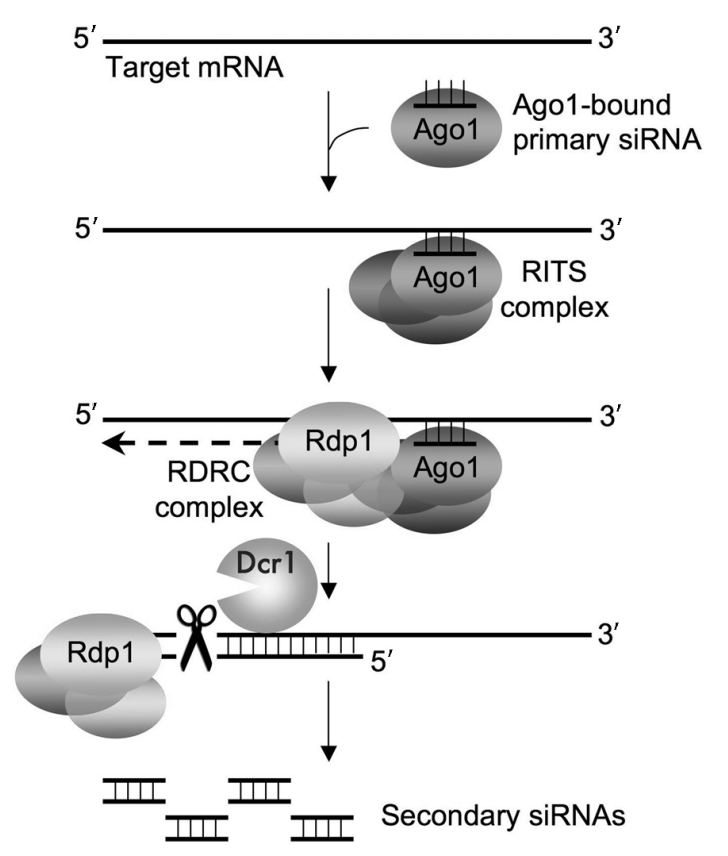

Figure 4. Secondary siRNA production in S. pombe. Agol loaded with a primary siRNA is targeted to centromeric chromatin via base pairing with a complementary nascent transcript. Once the Agol-containing RITS complex is engaged, it recruits the RNA-dependent RNA polymerase Rdp1 to the transcript as part of the RDRC complex. Rdp1 promotes primer-independent dsRNA synthesis without the need for Agol-mediated slicing of the transcript. Dcr1 ribonuclease activity associated with Rdp1 then processes the resulting long dsRNA into secondary siRNAs. 


\section{TETHERING CLR4 TO DNA BYPASSES RNAI TO ESTABLISH HETEROCHROMATIN}

Many studies, including the hairpin analyses described above, support the model whereby RNAi promotes H3K9 methylation by recruiting the HMT Clr4 to chromatin (Moazed 2009). A prediction of this model is that direct tethering of the chromatin-modifying machinery to DNA would render the RNAi pathway redundant for heterochromatin assembly. We sought to test this hypothesis by asking whether we could reconstitute heterochromatin at an otherwise euchromatic locus via artificial tethering of the Clr4 HMT (Kagansky et al. 2009). A DNA-binding domain from the Saccharomyces cerevisiae Gal4 protein (GBD) was fused to Clr4, and a reporter was created by inserting three Gal4-binding sites (3xgbs) upstream of $a d e 6^{+}$inserted at the ura 4 locus. Although full-length tethered Clr4 had no effect on ade $6^{+}$expression, tethering Clr4 lacking its chromodomain (GBD-Clr4- $\Delta$ cd) clearly resulted in silencing of the target $3 \times$ gbs-ade ${ }^{+}$locus, greatly reducing $a d e 6^{+}$expression (Kagansky et al. 2009). Deletion of the $\mathrm{Clr} 4$ chromodomain impairs its recruitment to endogenous heterochromatin and thus may release limiting factors to act at the tethering site. No silencing was observed following tethering of a Clr4 catalytic mutant (GBD-clr4- $\Delta \mathrm{cd}-\mathrm{H} 410 \mathrm{~K})$, indicating that silencing was directly dependent on the activity of tethered Clr4. Moreover, we detected H3K9me and Swi6 binding at the tethering site, consistent with formation of "synthetic" heterochromatin. The heterochromatin domain extended $5 \mathrm{~kb}$ on either side of the small ( $\sim 60 \mathrm{bp}$ ) tethering site, resulting in efficient silencing of genes flanking the $3 x g b s$ sequence (Kagansky et al. 2009). Thus, DNA-tethered Clr4 was sufficient to both modify histones in the vicinity of the tethering site and trigger propagation of heterochromatin along the chromosome arm (Fig. 5).

We next tested the requirement for RNAi in either establishment or maintenance of this synthetic heterochromatin. To test maintenance, we established GBD-clr4$\Delta \mathrm{cd}$-mediated silencing of the $3 x g b s-a d e 6^{+}$reporter in a wild-type background and then crossed out functional RNAi or chromatin components. Factors involved in RNAi were found to be dispensable for maintenance of preexisting silencing; this result is reminiscent of the modest part played by RNAi in the maintenance of heterochromatin at endogenous loci. We then asked whether silencing could also be established independently of RNAi by combining the Clr4-tether and reporter constructs directly in mutant backgrounds. Interestingly, and in contrast to what is seen at endogenous heterochromatic loci, establishment of silencing at the Clr4-tethered site is also RNAi independent. Thus, tethering Clr4 to DNA effectively bypasses the requirement for RNAi in heterochromatin formation. Conversely, both establishment and maintenance of synthetic heterochromatin-based silencing are dependent on chromatin factors including Chp2, Rik1 (Clr4 associated), and histone deacetylases - a prerequisite for $\mathrm{H} 3 \mathrm{~K} 9$ methylation. Surprisingly, despite associating with the synthetic heterochromatin, the chromodomain protein Swi6 does not actively participate in establishment or maintenance of the silencing process. This finding supports the recent suggestion that Swi6 contributes to silencing primarily through the RNAi-dependent processing of heterochromatic transcripts, possibly due to a role in anchoring nascent transcripts to chromatin (Motamedi et al. 2008). No siRNAs corresponding to $3 x g b s$-ade $6^{+}$were detected, supporting the idea that DNA-tethered $\mathrm{Clr} 4$ can form heterochromatin independently of RNAi. This system therefore provides a strong indication that RNAi principally functions upstream of Clr4 recruitment. However, Agol was enriched at the tethered locus, suggesting that the RITS complex can localize to synthetic heterochromatin via Chp1-mediated binding of $\mathrm{H} 3 \mathrm{~K} 9 \mathrm{me} 2$. By interlinking other components, Ago1 may have a structural role in heterochromatin integrity independent of RNAi activity.

We extended our study to address whether heterochromatin formed via tethered $\mathrm{Clr} 4$ can substitute for endogenous pericentromeric heterochromatin in establishing a functional centromere. A plasmid bearing only central domain DNA from a centromere cannot assemble a functional kinetochore; flanking heterochromatin is necessary for loading of the histone $\mathrm{H} 3$ variant CENP- $\mathrm{A}^{\text {cnp1 }}$ (Folco et al. 2008). However, a modified plasmid bearing $3 x g b s$ sites adjacent to the central domain was found to be mitotically stable in cells expressing the GBD-Clr4- $\Delta$ cd protein. Thus, synthetic heterochromatin is sufficient to promote loading of CENP- $\mathrm{A}^{\text {cnp1 }}$ on central core sequences and the assembly of a functional kinetochore (Fig. 5).

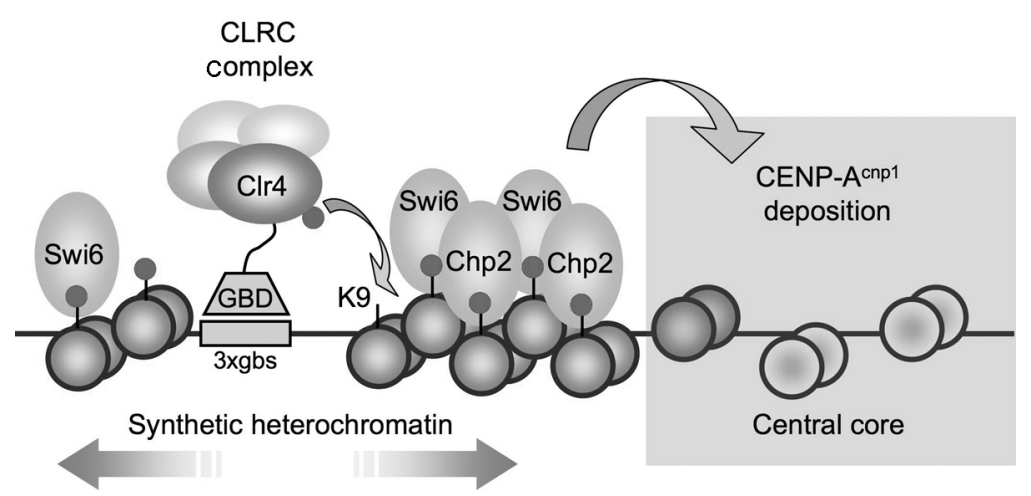

Figure 5. Synthetic heterochromatin-mediated CENP-A ${ }^{\text {cnp1 }}$ establishment. A GBDClr4 fusion protein tethered to three Gal4-binding sites (3xgbs) recruits the CLRC complex to chromatin. This promotes methylation of histone $\mathrm{H} 3$ on lysine 9 (K9), which is subsequently bound by chromodomain proteins Swi6 and Chp2. This synthetic heterochromatin spreads in cis over a $10-\mathrm{kb}$ region and, when assembled near centromeric central core sequences, promotes kinetochore formation via histone H3-variant CENP-A ${ }^{\text {cnp1 }}$ deposition. (Small solid dots) Methyl marks, (dark shaded circles) H3 nucleosomes, (light shaded circles) CENP-A ${ }^{\text {cnp } 1}$ nucleosomes. (Adapted from R. Almeida.) 
Dcr1 was dispensable for mitotic stability of the plasmid; synthetic heterochromatin can therefore substitute for both RNAi and centromeric outer-repeat sequences to support sister centromere cohesion and kinetochore assembly. Thus, neither the primary DNA sequence of the outer repeats nor the RNAi pathway is directly required for centromere function; rather, they are necessary only to direct heterochromatin formation, with the repeats providing a substrate for RNAi that serves to recruit $\mathrm{Clr} 4$ to chromatin.

\section{STC1 MEDIATES RNAI-DIRECTED HETEROCHROMATIN FORMATION}

Despite extensive characterization of RNAi and heterochromatin components in fission yeast, the mechanisms coupling the RNAi machinery to chromatin effectors have remained enigmatic. We have taken a genetic approach to identify missing pathway components that might bridge the gap between RNAi and chromatin modification. In a systematic screen of fission yeast strains bearing single gene deletions, we identified $s t c 1^{+}$, a novel gene required to maintain silencing of a marker gene inserted into the heterochromatic outer repeats of centromere 1 (Bayne et al. 2010). Cells lacking $s t c 1^{+}$mimic phenotypes of RNAi mutant cells: Centromeric siRNAs are undetectable, whereas moderate levels of $\mathrm{H} 3 \mathrm{~K} 9$ me are retained on the centromeric outer repeats. This contrasts with $c l r 4 \Delta$ cells, where $\mathrm{H} 3 \mathrm{~K} 9 \mathrm{me}$ is abolished, but a low level of siRNAs remains. Like other RNAi components, Stc1 is dispensable for maintenance of heterochromatin at the mating-type locus, and moreover, the Stc1 protein localizes to centromeric outer repeats and associates with noncoding centromeric transcripts (Bayne et al. 2010). Together, these observations strongly implicate Stc1 in the RNAi pathway.

To verify that Stc1 is required specifically for RNAidependent heterochromatin formation, we exploited the synthetic heterochromatin system described above. In this system, Clr4 artificially tethered to a euchromatic locus can establish and maintain heterochromatin assembly in an RNAi-independent manner. We predicted that if Stc1 functions in the RNAi pathway, its deletion should have no effect on this synthetic heterochromatin. As expected, like Dcr1, and in contrast to CLRC components, Stc1 proved to be dispensable for maintenance of synthetic heterochromatin. Surprisingly, however, tethered Clr4 could not establish silencing in the absence of Stc1. The requirement for Stc 1 to establish silencing in this system sets it apart from all other RNAi components tested and suggests a unique role for Stc1 that spans both RNAi and CLRC activities. This conclusion was supported by coimmunoprecipitation experiments, which showed that Stc1 associates with both the RNAi effector protein Agol and the chromatin-modifying complex CLRC. Together, these findings indicate that Stc1 acts at the interface between RNAi machinery and chromatin-modifying activities.

In wild-type cells, Stc1 association with Ago1 requires active RNAi, whereas the Stc1-CLRC interaction does not. These observations suggest a model whereby Stc1 mediates the RNAi-dependent recruitment of CLRC to chromatin. To test this hypothesis, we used a modified tethering system
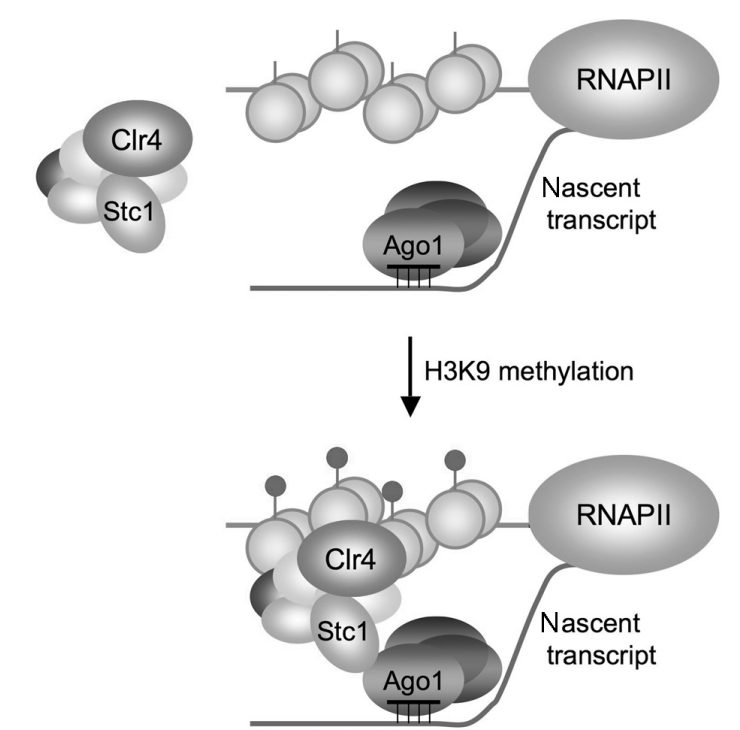

Figure 6. Stc1 connects RNAi to chromatin modification. Stc1 associates with the Clr4 HMT complex CLRC, independent of RNAi. When RNAi is active, the effector complex RITS (along with RDRC) is targeted to pericentromeric chromatin via Ago1bound siRNAs. Stc1 can then additionally associate with RITS on nascent pericentromeric transcripts, thereby mediating the RNAidependent recruitment of Clr4 to target chromatin and, hence, heterochromatin assembly. (Small solid circles) Methyl marks.

to determine whether tethering Stc1 to a euchromatic DNA region is sufficient to recruit chromatin modifiers independently of RNAi. Stc1 fused to a TetR ${ }^{\text {off }}$ DNA-binding domain (TetR ${ }^{\text {off-Stc } 1)}$ was recruited to a euchromatic ade $^{+}$ reporter gene via four adjacent tet operators (4xTetOade $\left.^{+}\right)$. Strikingly, tethered Stc1 was sufficient to induce $\mathrm{H} 3 \mathrm{~K} 9 \mathrm{me}$ at the target locus and silence ade $^{+}$expression. Maintenance of this tethered Stc1-mediated silencing was dependent on all CLRC components but was independent of the RNAi machinery; this assay demonstrates that, although critical for RNAi, Stc1 can recruit CLRC independently of RNA. Further supporting this conclusion, we found that point mutations affecting the LIM domain of Stc1 (K100A and R116A) disrupted the association of Stc1 with Ago1 but did not affect its interaction with CLRC components nor its ability to initiate heterochromatin formation when tethered to DNA. By separating chromatin modification from RNAi, this tethering assay reveals that Ago 1 binding and CLRC recruitment are distinct functions of Stc1, and both are required to facilitate heterochromatin assembly. These findings strongly support a model whereby Stc1 provides the connection between RNAi and chromatin modification: Agol engaged with nascent centromeric transcripts recruits Stc1, which in turn brings the CLRC complex to cognate chromatin to promote heterochromatin assembly (Fig. 6).

\section{CONCLUSIONS}

Studies in different model organisms point to an integrated role for RNAi machinery in the assembly of heterochromatin structures. Indeed, recent analyses have 
provided evidence of conserved links between siRNA production and $\mathrm{H} 3 \mathrm{~K} 9$ methylation in worms and vertebrates (Giles et al. 2010; Guang et al. 2010). Studies of fission yeast have provided detailed mechanistic insights into this process. However, codependency and positive feedback between RNAi and heterochromatin components have meant that the linear sequence of events that leads to heterochromatin assembly has remained unclear. The means by which cross talk occurs between RNAi and chromatin modification has also proven elusive.

We have used in vivo reconstitution assays to dissect the steps that lead to heterochromatin assembly. Using a hairpin construct as an artificial source of siRNAs, we were able to induce transcriptional gene silencing of homologous sequences in trans. However, heterochromatin assembly at the target locus was inefficient as compared with at the hairpin locus itself, supporting a strong cis-promoting mechanism in fission yeast. In the context of centromeric heterochromatin, this would suffice to efficiently induce chromatin modifications on outer-repeat sequences. How primary siRNAs are generated at centromeres remains an open question. However, an interesting possibility is that endogenous centromeric transcripts fold into natural hairpin structures that are processed by Dcr1 to generate siRNAs. Consistent with this idea, deep sequencing has identified two highly conserved siRNA clusters present at all three centromeres (Djupedal et al. 2009). These siRNAs are Rdp1 independent, as would be expected of primary siRNAs. Moreover, secondary structure probing indicates that transcripts from these regions are partially double stranded and can be processed by Dicer in vitro. Thus, the ability of centromeric noncoding RNAs to form natural hairpin structures may be sufficient to generate siRNAs and ultimately heterochromatin assembly in cis. Alternatively, a recent study identified Dcr1-independent small RNAs termed "primal small RNAs" (priRNAs) corresponding to the centromeric $d g$ repeats (Halic and Moazed 2010). These small RNAs are proposed to be degradation products of abundant transcripts that associate with Ago1. Bidirectional transcription across centromere repeats could allow priRNAs to target Agol to complementary nascent transcripts, nucleating siRNA production and H3K9 methylation. Whether Agol catalytic activity is required for priRNA biogenesis remains unclear. Interestingly, recent studies of mice and zebrafish have characterized a Dicer-independent RNAi pathway whereby Ago2-mediated cleavage of pre-microRNA (pre-miRNA) generates functional miRNAs (Cheloufi et al. 2010; Cifuentes et al. 2010).

Despite detailed investigations of the mechanism of RNAi-dependent heterochromatin formation, the molecular bridge connecting RNAi to chromatin modification has proven difficult to find. However, we have recently identified a new factor, Stc1, that appears to fulfill this role. Stc1 is a LIM-domain protein that interacts with both the RNAi effector protein Agol and the CLRC H3K9 methylation complex. Interestingly, a family of LIM-domain proteins was recently found to be required for miRNA-based silencing in human cells. These human LIM proteins interact with both Ago proteins and translation initiation complexes, thereby facilitating miRNA-me- diated translational repression of target transcripts (James et al. 2010). These findings suggest a conserved role for LIM-domain proteins in linking Ago-containing RNAi effector complexes to different gene regulation activities. Supporting the role of Stc1 as an RNAi-adapter molecule, DNA-tethered Stc1 can bypass RNAi and directly recruit CLRC to induce heterochromatin formation. In addition, experiments with DNA-tethered Clr4 suggest that Stc1 performs a further function in priming CLRC activity. How Stc1 "activates" the CLRC complex is still an open question. One possibility is that it relates to the E3 ubiquitin ligase activity of CLRC that has been demonstrated in vitro on histone H2B (Horn et al. 2005). Although the key substrates in vivo remain unclear, it may be that $\mathrm{H} 3 \mathrm{~K} 9$ methylation depends on prior histone ubiquitination, just as H2BK123 monoubiquitination promotes H3K79 methylation in S. cerevisiae (Ng et al. 2002). Such ubiquitination is potentially a key step in heterochromatin establishment that might also require Stc1.

Experiments with both DNA-tethered $\mathrm{Clr} 4$ and Stc1 demonstrate that when modifying enzymes are brought to chromatin via an alternative method, the requirement for RNAi can be bypassed. Importantly, synthetic heterochromatin formed in this way proved to be sufficient to establish a functional kinetochore on a plasmid-based minichromosome devoid of outer-repeat sequences. These findings indicate that the requirement for outer-repeat sequences and RNAi for CENP- $\mathrm{A}^{\text {cnp1 }}$ establishment and kinetochore assembly is fully explained by their role in nucleation of heterochromatin. In addition, these data support the model whereby RNAi principally functions upstream of $\mathrm{H} 3 \mathrm{~K} 9$ methylation in heterochromatin assembly. Other evidence for this comes from assays using minichromosomes bearing both central core and outer-repeat sequences, which show that, as at endogenous loci, RNAi is dispensable for maintenance of heterochromatin but is absolutely required for its establishment (Folco et al. 2008). Furthermore, it is now clear that H3K9 methylation is not essential for RNAi because low levels of siRNAs are detectable in both $c l r 4 \Delta$ (Bayne et al. 2010; Halic and Moazed 2010) and H3K9R mutant cells in which H3K9 cannot be methylated (Djupedal et al. 2009). Overall, existing data suggest that RNAi has a critical role in initiating H3K9 methylation, but once it has a foothold, H3K9me can propagate largely independently of RNAi. Nevertheless, robust heterochromatin likely requires positive feedback between both pathways, possibly involving Stc1 such that $\mathrm{H} 3 \mathrm{~K} 9$ me promotes further recruitment of RNAi to reinforce heterochromatin formation.

\section{ACKNOWLEDGMENTS}

We thank Alessia Buscaino for critical reading of the manuscript and members of the Allshire laboratory for helpful discussions. E.L. is supported by a Marie Curie Intra-European Fellowship within the European Community FP7; R.C.A is a Wellcome Trust Principal Research Fellow. E.H.B is an Medical Research Council Career Development Fellow. 


\section{REFERENCES}

Allen E, Xie Z, Gustafson AM, Carrington JC. 2005. microRNAdirected phasing during trans-acting siRNA biogenesis in plants. Cell 121: 207-221.

Allshire RC, Nimmo ER, Ekwall K, Javerzat JP, Cranston G. 1995. Mutations derepressing silent centromeric domains in fission yeast disrupt chromosome segregation. Genes Dev 9: 218-233.

Axtell MJ, Jan C, Rajagopalan R, Bartel DP. 2006. A two-hit trigger for siRNA biogenesis in plants. Cell 127: 565-577.

Bannister AJ, Zegerman P, Partridge JF, Miska EA, Thomas JO, Allshire RC, Kouzarides T. 2001. Selective recognition of methylated lysine 9 on histone $\mathrm{H} 3$ by the HP1 chromo domain. Nature 410: $120-124$.

Bartel DP. 2009. MicroRNAs: Target recognition and regulatory functions. Cell 136: 215-233.

Bayne EH, Portoso M, Kagansky A, Kos-Braun IC, Urano T, Ekwall K, Alves F, Rappsilber J, Allshire RC. 2008. Splicing factors facilitate RNAi-directed silencing in fission yeast. Science 322: 602-606.

Bayne EH, White SA, Kagansky A, Bijos DA, Sanchez-Pulido L, Hoe KL, Kim DU, Park HO, Ponting CP, Rappsilber J, et al. 2010. Stc1: A critical link between RNAi and chromatin modification required for heterochromatin integrity. Cell 140: 666 677.

Bühler M, Verdel A, Moazed D. 2006. Tethering RITS to a nascent transcript initiates RNAi- and heterochromatin-dependent gene silencing. Cell 125: 873-886.

Cheloufi S, Dos Santos CO, Chong MM, Hannon GJ. 2010. A dicer-independent miRNA biogenesis pathway that requires Ago catalysis. Nature 465: 584-589.

Chen ES, Zhang K, Nicolas E, Cam HP, Zofall M, Grewal SI. 2008. Cell cycle control of centromeric repeat transcription and heterochromatin assembly. Nature 451: 734-737.

Cifuentes D, Xue H, Taylor DW, Patnode H, Mishima Y, Cheloufi S, Ma E, Mane S, Hannon GJ, Lawson ND, et al. 2010. A novel miRNA processing pathway independent of Dicer requires Argonaute2 catalytic activity. Science 328: 1694-1698.

Colmenares SU, Buker SM, Bühler M, Dlakić M, Moazed D. 2007. Coupling of double-stranded RNA synthesis and siRNA generation in fission yeast RNAi. Mol Cell 27: 449-461.

Djupedal I, Kos-Braun IC, Mosher RA, Söderholm N, Simmer F, Hardcastle TJ, Fender A, Heidrich N, Kagansky A, Bayne E, et al. 2009. Analysis of small RNA in fission yeast; centromeric siRNAs are potentially generated through a structured RNA. EMBO J 28: 3832-3844.

Folco HD, Pidoux AL, Urano T, Allshire RC. 2008. Heterochromatin and RNAi are required to establish CENP-A chromatin at centromeres. Science 319: 94-97.

Ghildiyal M, Zamore PD. 2009. Small silencing RNAs: An expanding universe. Nat Rev Genet 10: 94-108.

Giles KE, Ghirlando R, Felsenfeld G. 2010. Maintenance of a constitutive heterochromatin domain in vertebrates by a Dicer-dependent mechanism. Nat Cell Biol (suppl. pp. 91-96) 12: 94-99.

Grewal SI, Elgin SC. 2007. Transcription and RNA interference in the formation of heterochromatin. Nature 447: 399-406.

Grewal SI, Jia S. 2007. Heterochromatin revisited. Nat Rev Genet 8: $35-46$.

Guang S, Bochner AF, Burkhart KB, Burton N, Pavelec DM, Kennedy S. 2010. Small regulatory RNAs inhibit RNA polymerase II during the elongation phase of transcription. Nature 465: 1097-1101.

Halic M, Moazed D. 2010. Dicer-independent primal RNAs trigger RNAi and heterochromatin formation. Cell 140: 504-516.

Hannon GJ. 2002. RNA interference. Nature 418: 244-251.

Hansen KR, Ibarra PT, Thon G. 2006. Evolutionary-conserved telomere-linked helicase genes of fission yeast are repressed by silencing factors, RNAi components and the telomere-binding protein Taz1. Nucleic Acids Res 34: 78-88.

Hong EJ, Villén J, Gerace EL, Gygi SP, Moazed D. 2005. A cullin E3 ubiquitin ligase complex associates with Rik1 and the Clr4 histone $\mathrm{H} 3-\mathrm{K} 9$ methyltransferase and is required for RNAi-mediated heterochromatin formation. RNA Biol 2: 106-111.
Horn PJ, Bastie JN, Peterson CL. 2005. A Rik1-associated, cullindependent E3 ubiquitin ligase is essential for heterochromatin formation. Genes Dev 19: 1705-1714.

Iida T, Nakayama J, Moazed D. 2008. siRNA-mediated heterochromatin establishment requires HP1 and is associated with antisense transcription. Mol Cell 31: 178-189.

James V, Zhang Y, Foxler DE, de Moor CH, Kong YW, Webb TM, Self TJ, Feng Y, Lagos D, Chu C-Y, et al. 2010. LIM-domain proteins, LIMD1, Ajuba, and WTIP are required for microRNAmediated gene silencing. Proc Natl Acad Sci 107: 1249912504.

Jenuwein T, Allis CD. 2001. Translating the histone code. Science 293: 1074-1080.

Jia S, Noma K, Grewal SI. 2004. RNAi-independent heterochromatin nucleation by the stress-activated ATF/CREB family proteins. Science 304: 1971-1976.

Jia S, Kobayashi R, Grewal SI. 2005. Ubiquitin ligase component Cul4 associates with Clr4 histone methyltransferase to assemble heterochromatin. Nat Cell Biol 7: 1007-1013.

Kagansky A, Folco HD, Almeida R, Pidoux AL, Boukaba A, Simmer F, Urano T, Hamilton GL, Allshire RC. 2009. Synthetic heterochromatin bypasses RNAi and centromeric repeats to establish functional centromeres. Science 324: 1716-1719.

Kanno T, Mette MF, Kreil DP, Aufsatz W, Matzke M, Matzke AJ. 2004. Involvement of putative SNF2 chromatin remodeling protein DRD1 in RNA-directed DNA methylation. Curr Biol 14: 801-805.

Kanoh J, Sadaie M, Urano T, Ishikawa F. 2005. Telomere binding protein Tazl establishes Swi6 heterochromatin independently of RNAi at telomeres. Curr Biol 15: 1808-1819.

Kloc A, Zaratiegui M, Nora E, Martienssen R. 2008. RNA interference guides histone modification during the $\mathrm{S}$ phase of chromosomal replication. Curr Biol 18: 490-495.

Lachner M, O'Carroll D, Rea S, Mechtler K, Jenuwein T. 2001. Methylation of histone $\mathrm{H} 3$ lysine 9 creates a binding site for HP1 proteins. Nature 410: 116-120.

Li F, Goto DB, Zaratiegui M, Tang X, Martienssen R, Cande WZ. 2005. Two novel proteins, Dos1 and Dos2, interact with Rik1 to regulate heterochromatic RNA interference and histone modification. Curr Biol 15: 1448-1457.

Luger K, Mäder AW, Richmond RK, Sargent DF, Richmond TJ. 1997. Crystal structure of the nucleosome core particle at 2.8 Å resolution. Nature 389: 251-260.

Matzke M, Kanno T, Daxinger L, Huettel B, Matzke AJ. 2009. RNA-mediated chromatin-based silencing in plants. Curr Opin Cell Biol 21: 367-376.

Moazed D. 2009. Small RNAs in transcriptional gene silencing and genome defence. Nature 457: 413-420.

Moissiard G, Parizotto EA, Himber C, Voinnet O. 2007. Transitivity in Arabidopsis can be primed, requires the redundant action of the antiviral Dicer-like 4 and Dicer-like 2, and is compromised by viral-encoded suppressor proteins. RNA 13: 12681278.

Motamedi MR, Verdel A, Colmenares SU, Gerber SA, Gygi SP, Moazed D. 2004. Two RNAi complexes, RITS and RDRC, physically interact and localize to noncoding centromeric RNAs. Cell 119: 789-802.

Motamedi MR, Hong EJ, Li X, Gerber S, Denison C, Gygi S, Moazed D. 2008. HP1 proteins form distinct complexes and mediate heterochromatic gene silencing by nonoverlapping mechanisms. Mol Cell 32: 778-790.

Nakayama J, Rice JC, Strahl BD, Allis CD, Grewal SI. 2001. Role of histone $\mathrm{H} 3$ lysine 9 methylation in epigenetic control of heterochromatin assembly. Science 292: 110-113.

$\mathrm{Ng} \mathrm{HH}$, Xu RM, Zhang Y, Struhl K. 2002. Ubiquitination of histone H2B by Rad6 is required for efficient Dot1-mediated methylation of histone H3 lysine 79. J Biol Chem 277: 3465534657.

Pak J, Fire A. 2007. Distinct populations of primary and secondary effectors during RNAi in C. elegans. Science 315: 241-244.

Passarge E. 1979. Emil Heitz and the concept of heterochromatin: Longitudinal chromosome differentiation was recognized fifty years ago. Am J Hum Genet 31: 106-115. 
Pidoux AL, Allshire RC. 2005. The role of heterochromatin in centromere function. Philos Trans R Soc Lond B Biol Sci 360: 569579.

Sadaie M, Iida T, Urano T, Nakayama J. 2004. A chromodomain protein, Chp1, is required for the establishment of heterochromatin in fission yeast. $E M B O J$ 23: 3825-3835.

Sigova A, Rhind N, Zamore PD. 2004. A single Argonaute protein mediates both transcriptional and posttranscriptional silencing in Schizosaccharomyces pombe. Genes Dev 18: 2359-2367.

Sijen T, Fleenor J, Simmer F, Thijssen KL, Parrish S, Timmons L, Plasterk RH, Fire A. 2001. On the role of RNA amplification in dsRNA-triggered gene silencing. Cell 107: 465-476.

Sijen T, Steiner FA, Thijssen KL, Plasterk RH. 2007. Secondary siRNAs result from unprimed RNA synthesis and form a distinct class. Science 315: 244-247.

Simmer F, Buscaino A, Kos-Braun IC, Kagansky A, Boukaba A, Urano T, Kerr AR, Allshire RC. 2010. Hairpin RNA induces secondary small interfering RNA synthesis and silencing in trans in fission yeast. EMBO Rep 11: 112-118.

Sugiyama T, Cam H, Verdel A, Moazed D, Grewal SI. 2005. RNAdependent RNA polymerase is an essential component of a selfenforcing loop coupling heterochromatin assembly to siRNA production. Proc Natl Acad Sci 102: 152-157.

Vaistij FE, Jones L, Baulcombe DC. 2002. Spreading of RNA targeting and DNA methylation in RNA silencing requires transcription of the target gene and a putative RNA-dependent RNA polymerase. Plant Cell 14: 857-867.

Verdel A, Jia S, Gerber S, Sugiyama T, Gygi S, Grewal SI, Moazed D. 2004. RNAi-mediated targeting of heterochromatin by the RITS complex. Science 303: 672-676.

Volpe TA, Kidner C, Hall IM, Teng G, Grewal SI, Martienssen RA. 2002. Regulation of heterochromatic silencing and histone H3 lysine-9 methylation by RNAi. Science 297: 1833-1837.

Zhang K, Mosch K, Fischle W, Grewal SI. 2008. Roles of the Clr4 methyltransferase complex in nucleation, spreading and maintenance of heterochromatin. Nat Struct Mol Biol 15: 381-388. 


\section{$\$_{\mathrm{CSH}}^{\infty}$ Cold Spring Harbor Symposia SYMPOSIA on Quantitative Biology}

\section{On the Connection between RNAi and Heterochromatin at Centromeres}

E. Lejeune, E.H. Bayne and R.C. Allshire

Cold Spring Harb Symp Quant Biol 2010 75: 275-283 originally published online February 2, 2011 Access the most recent version at doi:10.1101/sqb.2010.75.024

References This article cites 56 articles, 20 of which can be accessed free at: http://symposium.cshlp.org/content/75/275.full.html\#ref-list-1

\section{License}

Email Alerting Receive free email alerts when new articles cite this article - sign up in the box at the Service top right corner of the article or click here. 